\title{
Exploring the low-energy domain of LAT-detected GRBs
}

\author{
Elisabetta Bissaldi* \\ Dipartimento Interateneo di Fisica, Politecnico di Bari, 70125 Bari, Italy; \\ INFN - Sezione di Bari, Bari, Italy \\ E-mail: elisabetta.bissaldieba.infn.it
}

\section{Nicola Omodei}

W. W. Hansen Experimental Physics Laboratory, Kavli Institute for Particle Astrophysics and Cosmology, Department of Physics and SLAC National Accelerator Laboratory, Stanford University, Stanford, CA 94305, USA

\section{Giacomo Vianello}

W. W. Hansen Experimental Physics Laboratory, Kavli Institute for Particle Astrophysics and Cosmology, Department of Physics and SLAC National Accelerator Laboratory, Stanford University, Stanford, CA 94305, USA

\section{Daniel Kocevski}

Astrophysics Office, ST12, NASA/Marshall Space Flight Center, Huntsville, AL 35812, USA

\section{on behalf of the Fermi-LAT Collaboration}

In preparation for the Second LAT Gamma-Ray Burst (GRB) catalog, we explore the use of the LAT low-energy (LLE) data selection to detect bursts with gamma-ray energy below $100 \mathrm{MeV}$. Here we present the sample of GRBs that are detected at these energies through a Bayesian Block analysis of all Fermi Gamma-ray Burst Monitor (GBM) triggers collected in 8 years of Fermi mission. In particular, we compare the temporal characteristics of this sample of GRBs with those of the brightest bursts detected above $1 \mathrm{MeV}$ by the GBM. Finally, we examine the properties of a subset of events which are not detected above $100 \mathrm{MeV}$ (LLE-only GRBs).

7th Fermi Symposium 2017

15-20 October 2017

Garmisch-Partenkirchen, Germany

\footnotetext{
*Speaker.
} 


\section{Introduction}

Since 2008, the synergy between the two instruments on board the Fermi mission has been providing valuable information in order to better understand the high-energy emission mechanisms of GRBs. The Gamma-Ray Burst Monitor (GBM) has been constantly monitoring the full sky unocculted by the Earth. It already detected more than 2200 bursts during the first 9.5 years of operation. GBM consists of two types of detectors, namely $12 \mathrm{NaI}$ detectors (8-900 keV) and 2 BGO detectors $(250 \mathrm{keV}-40 \mathrm{MeV})$. The main temporal and spectral properties of the detected GRBs are summarized in the official Fermi-GBM catalogs [1, 2, 3].

The primary instrument on-board Fermi, the Large Area Telescope (LAT), which detects gamma rays above $100 \mathrm{MeV}$ up to more than $300 \mathrm{GeV}$, has also been active in catching GRBs at such high energies. The first GRB catalog [4] included 35 GRBs and highlighted their main high-energy properties. As it was immediately evident, almost $70 \%$ of these bursts were detected by LAT also below $100 \mathrm{MeV}$. This detection was made possible by applying a non-standard analysis reconstruction, the so-called "LAT Low-Energy" (LLE) technique [5].

When compared to the standard LAT "P8 TRANSIENT020E" data [6], LLE data are produced with looser selection criteria. In particular, it is required that the selected events have at least one track detected in the LAT tracker, i.e. have a reconstructed direction together with the best estimation of the reconstructed energy. The effective area below $100 \mathrm{MeV}$ and at larger off-axis angles $\left(>60^{\circ}\right)$ is also larger. Unfortunately, LLE data exhibit a higher background contamination and limited spatial resolution. Therefore, it is basically designed for the analysis of gamma-ray transients (GRBs, solar flares [7]) with soft spectra or occurring at high off-axis angles. It allows detailed studies of the temporal structure of GRB emission and it is of particular interest for analyzing short transients. In the framework of the LAT GRB catalog, LLE data is mainly used for source detection and duration measurements.

\section{A new LLE analysis}

Since 2013, the LAT analysis tools have been greatly improved, mainly thanks to the transition to the Pass 8 event-level reconstruction. Moreover, the LAT collaboration implemented a new GRB-detection scheme, namely the LAT Transient Factory (LTF [8]). The application of this tool made it possible to overcome the large GBM systematic error on the localization by search the LAT data on different time scales and on larger regions of the sky. The increase in the rate of LAT-detected bursts is of the order of $\sim 50 \%$, which became evident also from the number of circulars publicly distributed to the Gamma-Ray Coordinate Network (GCNs). The second LAT GRB catalog is currently in preparation within the LAT collaboration and includes more than 130 bursts.

Regarding the LLE data, over the last years we developed a new algorithm, namely a modified version of the Bayesian Block analysis $[9,10]$ which takes into account the presence of a timevarying background. The advantages of applying such a method to LLE data are that it is time-scale agnostic, it has built-in a trial factor correction and it is computationally very fast.

The algorithm defines a first "search" window around the trigger time and two "off-pulse" windows, covering some hundreds of seconds before and after the trigger time. The background is 
then fitted with a polynomial function, which is multiplied by the cosine of the LAT zenith angle with respect to the source location in the sky. In this step, a least square fit of all non-zero bins is first carried out, and then it is refined by using Poisson log-likelihood including all bins. The background goodness of fit is computed with simulations. We then applied the modified Bayesian Block (modBB) algorithm to the "search" window, and in case of detection, we measure the net flux, providing a significance value that takes into account the background uncertainty.

\section{Preliminary results}

We tested the algorithm on all GBM-detected GRBs over a period of 9 years (from August 2008 to July 2017) and found that $\sim 80$ GRBs show significant emission below $100 \mathrm{MeV}$, which we refer to as the "LLE sample". These represent $\sim 60 \%$ of all GRBs detected with the standard LAT analysis above $100 \mathrm{MeV}$. Moreover, $\sim 15 \%$ of the LLE sample shows no emission at higher energies. We refer to this fraction of bursts as the "LLE-only" sample.

The smaller fraction of LLE bursts compared to the one described in the first LAT GRB catalog $(\sim 70 \%)$ can be attributed to the overall larger number of bursts retrieved with the standard analysis, which largely improved thanks to the transition to Pass 8 and to the LTF features. Finally, The LLEonly sample comprises mainly long GRBs, with only $\sim 15 \%$ being short GRBs. Final numbers are currently under revision and will be published in the second LAT GRB catalog [8].

\subsection{Temporal properties}

We studied the temporal characteristics of the bursts in the LLE sample, calculated in the 10$100 \mathrm{MeV}$ energy range, and compared them to the same ones calculated at lower energies with
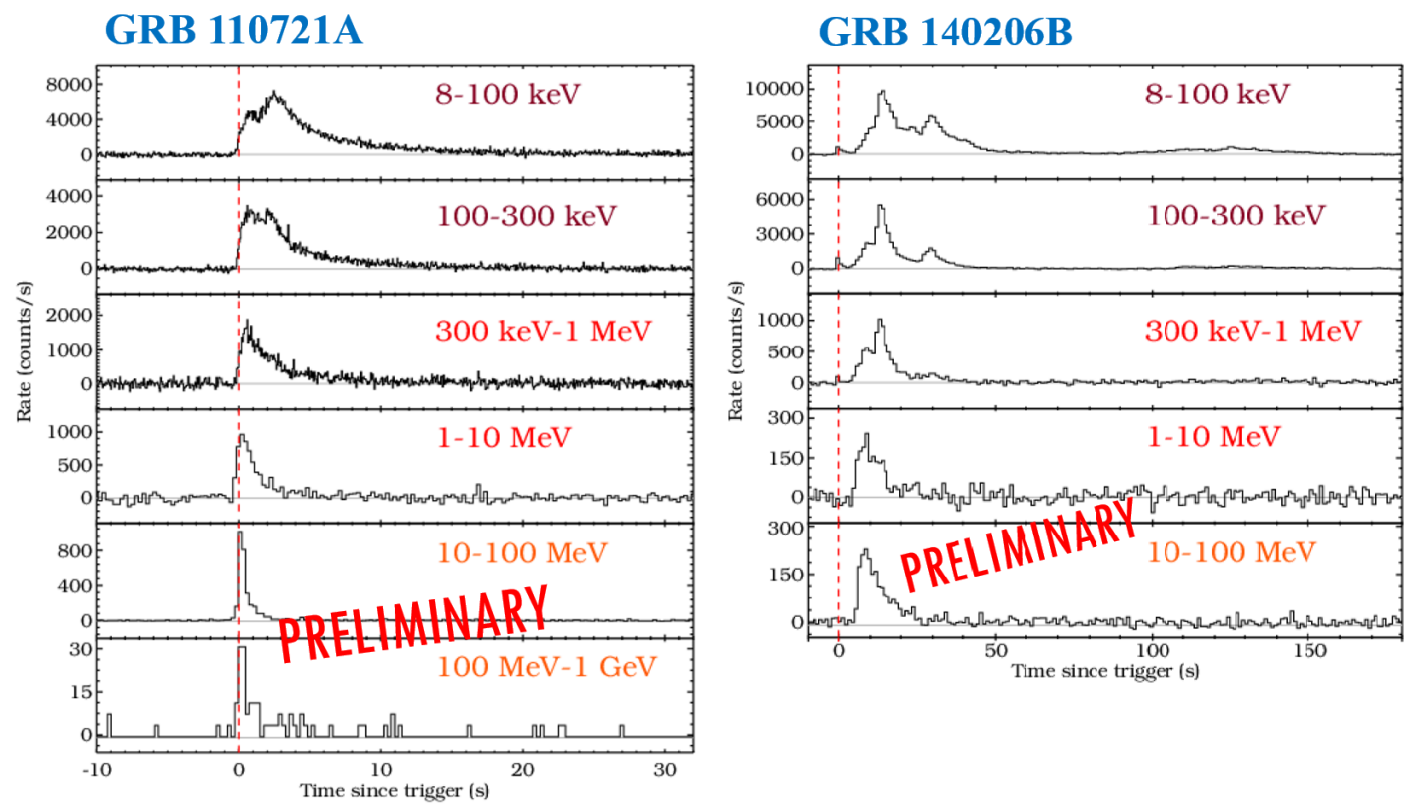

Figure 1: Lightcurves of two LAT-detected long GRBs, GRB 110721A (left plot) and GRB 140206B (right plot), observed at different energies. 

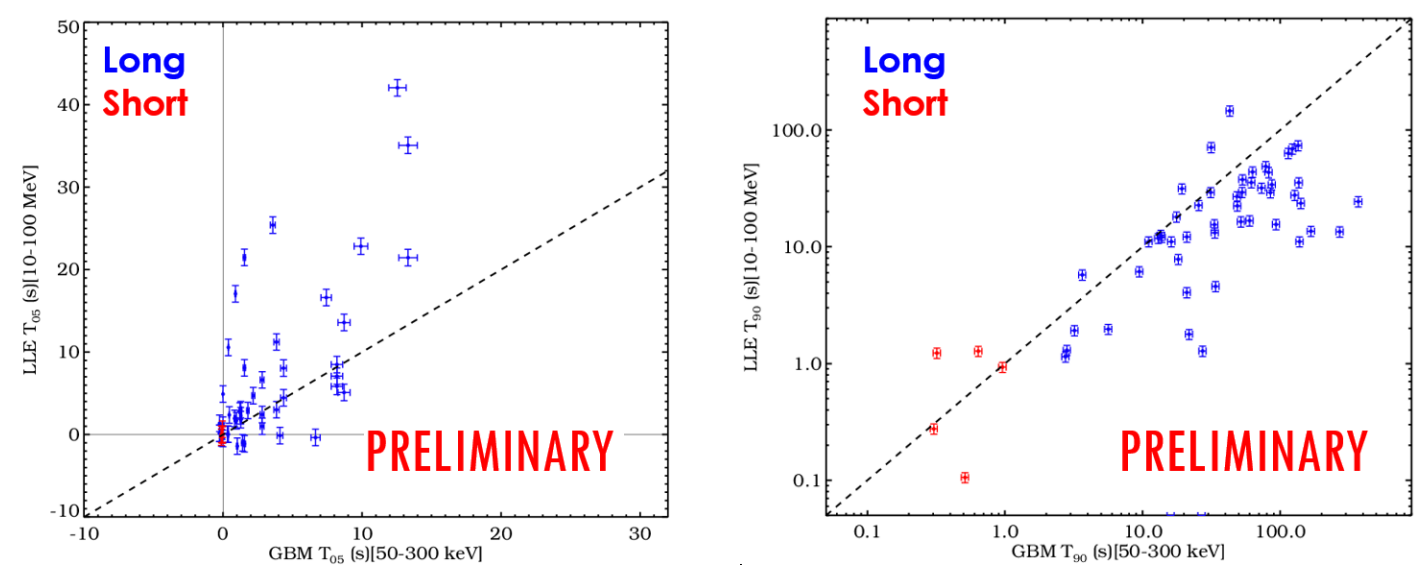

Figure 2: Distribution of $T_{05}$ and $T_{90}$ measured in the $10-100 \mathrm{MeV}$ energy range versus the same quantities measured in the 50-300 keV energy range.

GBM data, in the 50-300 keV energy band. We first explored how the GRB lightcurve evolves at different energies, as displayed in Figure 1. The two top panels of each plot represent low-energy data as measured by the GBM NaI detectors from 8 to $300 \mathrm{keV}$. The subsequent two panels show emission measured by the GBM BGO detectors in the energy range from $300 \mathrm{keV}$ to $10 \mathrm{MeV}$. The bottom panel(s) show(s) the high-energy emission detected by the LAT. If the burst an "LLE-only" one, then just one panel is shown, with data ranging from 10 to $100 \mathrm{MeV}$, as in the case of GRB $140206 \mathrm{~B}$ (see the plot on the right). If the burst is observed also in the LAT standard energy range, the emission in the $100 \mathrm{MeV}$ to $1 \mathrm{GeV}$ lightcurve is shown, too, as in the case of GRB $110721 \mathrm{~A}$ (see the plot on the left).

Figure 2 shows the distribution of the onset times (the so-called $T_{05}$, left plot) and the duration (the so-called $T_{90}$, right panel) in the high-energy range versus the same quantities measured in the low-energy range, the dotted line indicating equivalence. It can be seen that most of the bursts in the LLE sample have a similar onset time at higher energies as at lower energies, with just a fraction starting significantly later. Durations tend to be systematically shorter at higher energies, as it is also clearly visible in the lightcurves of the two example bursts shown in Figure 1.

The temporal properties of the LLE sample GRBs reprensent an interesting dataset in order to study the relationship between the pulse paradigm at high energies and the luminosity distribution in GRBs [11]. Moreover, the duration inferred from the LLE data analysis can provide important information for those extremely bright events, as in the case of GRB 130427A [12].

\section{Future steps}

With this preliminary analysis, we showed that by exploring the energy range below $100 \mathrm{MeV}$ we can significantly contribute to the second LAT GRB catalog catalog, currently in preparation. In particular, the inclusion of the LLE-only sample adds almost $10 \%$ to the final number of LATdetected bursts. This will allow us to further investigate interesting GRB features which could be hidden at $\mathrm{MeV}$ energies, as shown also in [10]. In the near future, we plan to refine the LLE duration measurements and to study the duration versus energy relations of the LLE sample. 


\section{Acknowledgments}

The Fermi-LAT Collaboration acknowledges support for LAT development, operation and data analysis from NASA and DOE (United States), CEA/Irfu and IN2P3/CNRS (France), ASI and INFN (Italy), MEXT, KEK, and JAXA (Japan), and the K.A. Wallenberg Foundation, the Swedish Research Council and the National Space Board (Sweden). Science analysis support in the operations phase from INAF (Italy) and CNES (France) is also gratefully acknowledged. This work performed in part under DOE Contract DE-AC02-76SF00515. E.B. ackowledges the Italian "Fondo di Sviluppo e Coesione 2007-2013 - APQ Ricerca Regione Puglia - Future In Research".

\section{References}

[1] P. N. Bhat, et al., The Third Fermi GBM Gamma-Ray Burst Catalog: The First Six Years ApJSS 223, 2,28 (2016)

[2] D. Gruber, et al., The Fermi GBM Gamma-Ray Burst Spectral Catalog: Four Years of Data, ApJSS 211, 1, 12 (2014)

[3] H.-F. Yu, et al., The Fermi GBM gamma-ray burst time-resolved spectral catalog: brightest bursts in the first four years, A\&A 588, 135 (2016)

[4] M. Ackermann, et al., The First Fermi-LAT Gamma-Ray Burst Catalog ApJSS 209, 1, 11 (2013)

[5] V. Pelassa, et al., The LAT Low-Energy technique for Fermi Gamma-Ray Bursts spectral analysis, Fermi Symposium eConf Proceedings C091122 (2009) [11002.2617]

[6] https://fermi.gsfc.nasa.gov/ssc/data/access/lat/

[7] M. Ajello, et al., Impulsive and Long Duration High-energy Gamma-Ray Emission from the Very Bright 2012 March 7 Solar Flares ApJ 789, 1, 20 (2014)

[8] G. Vianello, et al., The First 100 LAT Gamma-Ray Bursts: A New Detection Algorithm and Pass 8, Fermi Symposium proceedings C141020.1 (2015)

[9] J. D. Scargle, et al., Studies in Astronomical Time Series Analysis. VI. Bayesian Block Representations, ApJ 764, 2 (2013)

[10] G. Vianello, et al., The Bright and the Slow - GRBs 100724B \& 160509A with high-energy cutoffs at lessim100; MeV, submitted to ApJ (2017)

[11] J. P. Norris, et al., Connection between Energy-dependent Lags and Peak Luminosity in Gamma-Ray Bursts ApJ 5341 (2000)

[12] M. Ackermann, et al., Fermi-LAT Observations of the Gamma-Ray Burst GRB 130427A Science 343 6166 (2014) 